\title{
To Principles of Quantum Theory Construction
}

\author{
Dmitri Yerchuck ${ }^{1, *}$, Alla Dovlatova ${ }^{2}$, Andrey Alexandrov ${ }^{2}$ \\ ${ }^{1}$ Heat-Mass Transfer Institute of National Academy of Sciences of RB, Brovka Str.15, Minsk \\ ${ }^{2}$ M.V.Lomonosov Moscow State University, Moscow \\ *Corresponding author: dpy@tut.by
}

Received October 09, 2014; Revised October 23, 2014; Accepted October 26, 2014

\begin{abstract}
New insight to the principles of the quantum theory construction is given. It is based on the symmetry study of main differential equations of mechanics and electrodynamics. It has been shown, that differential equations, which are invariant under transformations of groups, which are symmetry groups of mathematical numbers (considered within the frames of the number theory) determine the mathematical nature of the quantities, incoming in given equations. The substantial consequence of the given consideration is the proof of the main postulate of quantum mechanics, that is, the proof of the statement, that to any quantum mechanical quantity can be set up into the correspondence the Hermitian matrix. It is shown, that a non-abelian character of the multiplicative group of the quaternion ring leads to the nonapplicability of the quaternion calculus for the construction of new versions of quantum mechanics directly. The given conclusion seems to be actual, since there is a number of modern publications with the development of the quantum mechanics theory using the quaternions with the standard basis $\{\mathrm{e}$, $\mathrm{i}, \mathrm{j}, \mathrm{k}\}$. The correct way for the construction of new versions of quantum mechanics on the quaternion base is discussed in the paper presented. It is realized by means of the representation of the quaternions through the basis of the linear space of complex numbers over the field of real numbers, under the multiplicative group of which the equations of the dynamics of mechanical systems are invariant. At the same time the quaternion calculus is applicable in electrodynamics, at that the new versions of quantum electrodynamics can be constructed by an infinite number of the ways corresponding to an infinite number of the matrix representations of the standard quaternion basis $\{\mathrm{e}, \mathrm{i}, \mathrm{j}, \mathrm{k}\}$. The given conclusion is the consequence of the high symmetry of Maxwell equations.
\end{abstract}

Keywords: symmetry, differential equations, quantum theory, quaternions

Cite This Article: Dmitri Yerchuck, Alla Dovlatova, and Andrey Alexandrov, "To Principles of Quantum Theory Construction.” International Journal of Physics, vol. 2, no. 6 (2014): 181-188. doi: 10.12691/ijp-2-6-1.

\section{Introduction}

The onrush of the nanoelectronics, spintronics the successes in the elaboration of logic quantum systems including quantum computers and quantum communication systems require the corresponding development of the quantum theory. To develop the quantum theory it seems to be necessary to have a rather deep insight into the principles of its construction. It is well known, that the quantum theory is formulated by using of some postulates. We will concern in the work presented the only of the main postulate, from which is begun the study of quantum mechanics in all textbooks (see, for example, [1,2,3,4]). Its formulation is the following: "The linear selfadjoint operators are setting up into correspondence to observable physical quantities". It is the first main postulate of quantum mechanics. It seems to be interesting, why it was introduced by originators and how much is its fundamentality. In other words, is it the corner stone, the introduction of which cannot be grounded, or it is the consequence of more general laws of the Nature, which were not taken into account by founding fathers of the quantum theory and their followers. In the given connection let us concern the history of the introduction of the given post late in the theory. The first main postulate is really contained in the first works on quantum mechanics, which are referred to be the birth of quantum mechanics, that is in the works of Heisenberg, Born, Jordan, Dirac, Pauli. The mathematical basis of Heisenberg's treatment [5] is the law of the multiplication of quantum-theoretical quantities, which he derived from an ingenious consideration of correspondence arguments. The development of his formalism, which has been given in the paper of Born and Jordan [6], is based upon the fact that the rule of the multiplication is none other than the well-known mathematical rule of the matrix multiplication. The infinite square array (with discrete or continuous indices) termed a matrix, is a representation of a physical quantity which is given in the classical theory being to be a function of time. The mathematical method of the treatment, which was accepted to be inherent for the quantum mechanics, is thereby characterized through the employment of the matrix analysis instead of the usual number analysis [6]. At the same time, it is clear, that matrices realise the representation of corresponding operators in the corresponding to their dimensionality space. Moreover, it was directry formulated in [6], that the matrices, which are setting up in the correspondence to observable physical quantities have to be Hermitian 
matrices. It is given in [6] on the concrete example of the description of the dynamic system.

The arguments for the introduction of the first main postulate were determined in fact mainly by the possibility to explain the experimental data, which could not be explained by the classical theory. Ones of the first experimental data, which were explained, using the first main postulate, were spectroscopic data, obtained from the experimental absorption studies of the hydrogen atoms. The quantum physics interpretation of the spectrum of the hydrogen atom has been done for the first time by Pauli [9]. The significant moment in the explanation of the correctness of the introduction of the given postulate was the connection of quantum mechanics with classical mechanics. The requirement of the connection of quantum mechanics with classical mechanics is especially characteristic for the work of Dirac [8]. Dirac rediscovered and developed in the paper cited the main ideas of the Born-Jordan paper. For instance, starting with classical mechanics, Dirac formulates the quantum equation of the motion for any quantity

$$
\frac{d x}{d t}=[x, \mathrm{H}]
$$

where $\mathrm{H}$ is Hamiltonian.

The quantum mechanics foundations underlying in the papers [5,6] were developed in [7] for the description of systems having arbitrarily many-degrees of freedom using the same main postulate.

It seems to be interesting the following moment. In Heisenberg's fundamental paper and in the papers $[6,7,8,9]$ following it, dynamical variables were assumed to be represented by matrices. However in the paper [10], Dirac starts with a more general assumption. He supposes the quantum variables $\mathrm{x}, \mathrm{y}, \ldots$ to be elements of an algebra, which means that sums $\mathrm{x}+\mathrm{y}$ and products $\mathrm{xy}$ are defined in the way, which satisfy the ordinary laws of algebra, excluding the commutative law of the multiplication. There are among the elements of the algebra proposed by Dirac the numbers of the classical mathematics which were called c-numbers. All other elements were called qnumbers. It was in fact the step to the proof of the first main postulate (see further), although the attempt to proof the given postulate was not undertaken from the birth of quantum mechanics in 1925 up to now. We have shown in the recent brief Conference report [11], that the given postulate can be strictly proved.

The aim of the paper presented is to give more detailed description of the results, reported in [11] and to develop the concept described in the given work. In particular, the possibility of the application of the quaternion calculus to quantum mechanics is discussed.

\section{Connection of Symmetry of Quantum Systems with Algebra of Complex Numbers}

A number of useful results from the algebra of complex numbers, being to be applicable to quantum theory were summarized in the paper [14]. They will be reproduced here for the convenience of the readers and will be supplemented with some results from the algebra of the hypercomplex numbers, which seem also to be significant in applications to tasks of quantum physics.

It is well known, that the basis of the linear space of complex numbers over the field $\mathrm{R}$ of real numbers is choosed usually being to be consisting of the numbers 1 and $\mathrm{i}$. At the same time to any complex number $\mathrm{a}+\mathrm{ib}$, a,b $\in \mathrm{R}$, represented in the given basis, can be set up in conformity the [2x2]-matrix according to biective mapping

$$
f: a+i b \rightarrow\left[\begin{array}{cc}
a & -b \\
b & a
\end{array}\right]
$$

From the bijectivity of mapping (2) follows the existence of inverse mapping. It means that to any matrix, which has the structure, given by the right side in the relation (2), corresponds the complex number, determined by the left side. The matrices

$$
\left[\begin{array}{ll}
1 & 0 \\
0 & 1
\end{array}\right],\left[\begin{array}{cc}
0 & -i \\
i & 0
\end{array}\right]
$$

produce the basis for complex numbers $\{a+i b\}, a, b \in R$ in the linear space of [2x2]-matrices, defined over the field of real numbers.

It is argued in above cited work, that it is convenient often to define the space of complex numbers over the group $\mathrm{Z}$ of real positive numbers including zero. In the given case the dimensionality of the matrices and their basis has to be duplicated, since to two unities - positive 1 and negative $\mathrm{i} 1$ can be set up in conformity the [2x2]matrices according to the following biective mapping

$$
\xi: 1 \rightarrow\left[\begin{array}{ll}
1 & 0 \\
0 & 1
\end{array}\right],-1 \rightarrow\left[\begin{array}{ll}
0 & 1 \\
1 & 0
\end{array}\right]
$$

It is evident, that the relation 4 allows to recreate the operations with negative numbers without recourse of negative numbers themselves. So, the following [4x4]matrices, so called $[0,1]$-matrices,

$$
\begin{aligned}
\zeta: 1 \rightarrow\left[e_{1}\right] & =\left[\begin{array}{llll}
1 & 0 & 0 & 0 \\
0 & 1 & 0 & 0 \\
0 & 0 & 1 & 0 \\
0 & 0 & 0 & 1
\end{array}\right], i \rightarrow\left[e_{2}\right]=\left[\begin{array}{llll}
0 & 1 & 0 & 0 \\
0 & 0 & 1 & 0 \\
0 & 0 & 0 & 1 \\
1 & 0 & 0 & 0
\end{array}\right], \\
-1 \rightarrow\left[e_{3}\right] & =\left[\begin{array}{llll}
0 & 0 & 1 & 0 \\
0 & 0 & 0 & 1 \\
1 & 0 & 0 & 0 \\
0 & 1 & 0 & 0
\end{array}\right],-i \rightarrow\left[e_{4}\right]=\left[\begin{array}{llll}
0 & 0 & 0 & 1 \\
1 & 0 & 0 & 0 \\
0 & 1 & 0 & 0 \\
0 & 0 & 1 & 0
\end{array}\right] .
\end{aligned}
$$

can also be the basis of the space of complex numbers over the group $\mathrm{Z}$ of real positive numbers (including zero).

We see from the given example, that the choise of the basis is ambiguous. Any four [4x4] [0,1]-matrices, which satisfy the rules of the cyclic recurrence

$$
i^{1}=i, i^{2}=-1, i^{3}=-1, i^{4}=1
$$

can be the basis of the basis of the space of complex numbers over the same group $\mathrm{Z}$ of real positive numbers (including zero). In particular, the following [4x4] [0,1]matrices 


$$
\begin{aligned}
& {\left[e_{1}^{\prime}\right]=\left[\begin{array}{llll}
1 & 0 & 0 & 0 \\
0 & 1 & 0 & 0 \\
0 & 0 & 1 & 0 \\
0 & 0 & 0 & 1
\end{array}\right],\left[e_{2}^{\prime}\right]=\left[\begin{array}{llll}
0 & 0 & 1 & 0 \\
0 & 0 & 0 & 1 \\
0 & 1 & 0 & 0 \\
1 & 0 & 0 & 0
\end{array}\right],} \\
& {\left[e_{3}^{\prime}\right]=\left[\begin{array}{llll}
0 & 1 & 0 & 0 \\
1 & 0 & 0 & 0 \\
0 & 0 & 0 & 1 \\
0 & 0 & 1 & 0
\end{array}\right],\left[e_{4}^{\prime}\right]=\left[\begin{array}{llll}
0 & 0 & 0 & 1 \\
0 & 0 & 1 & 0 \\
1 & 0 & 0 & 0 \\
0 & 1 & 0 & 0
\end{array}\right] .}
\end{aligned}
$$

can also be the basis of the complex numbers' linear space. It is evident, that the set of [0,1]-matrices, given by (7), is isomorphous to the set, which is given by (5).

Thus, we come to the conclusion, that the system of complex numbers can be constructed by the infinite number of the ways, at that, the cyclic basis can consist of $\mathrm{m}$ units, $\mathrm{m} \in \mathrm{N}$, starting from three.

It is remarkable, that the conformity between complex numbers and matrices is realized by biective mappings. It means, that inverse mapping is also exists. Consequently, to any squarte matrix, belonging to the linear space with a basis given by (5), or (7), or any other, satisfying the rules of the cyclic recurrence like to (6) can be set up in conformity the complex number.

For the applications in quantum physics is especially significant, that to any Hermitian matrix $\mathrm{H}$ can be set up in conformity the following complex number

$$
\zeta: H \rightarrow S+i A=\left[\begin{array}{cc}
S & -A \\
A & S
\end{array}\right]
$$

where S and A are symmetric and ant symmetric parts of a Hermitian matrix.

A number of rather complicated tasks in quantum physics can be solved by an application of a hypercomplex n-numbers' calculus. The apparatus of hypercomplex n-numbers was used successfully, for instance, by the calculation of optical properties of $1 \mathrm{D}$ carbon zigzag shaped nanotubes, taking into account the quantum nature of EM-field in the works [12,13].

Hypercomplex n-numbers are defined in the hypercomplex n-numbers' calculus to be the elements of the commutative ring $\mathrm{Zn}$, being to be the direct sum of $n$ fields $\mathrm{C}$ of complex numbers, $\mathrm{n} \in \mathrm{N}$, that is

$$
Z_{n}=C \oplus C \oplus \ldots \oplus C \text {. }
$$

It is seen from 9, that any hypercomplex n-number $\mathrm{z} \in$ $\mathrm{Z}_{\mathrm{n}}$ is $\mathrm{n}$-dimensional quantity with the components $\mathrm{k}_{\alpha} \in \mathrm{C}$, $\alpha=\overline{0, n-1}$. Its representation in the row matrix form is

$$
z=\left|k_{\alpha}\right|=\left|k_{0}, k_{1}, \ldots, k_{n-1}\right|,
$$

where $z \in Z_{n}$. On the other hand, hypercomplex n-number $\mathrm{z} \in \mathrm{Z}_{\mathrm{n}}$ can also be represented in the following form

$$
z=\sum_{\alpha=0}^{n-1} k_{\alpha} \pi_{\alpha}
$$

where the set $\left\{\pi_{\alpha}\right\}, \alpha=\overline{0, n-1}$ is the basis of $Z_{n}$. Basis elements of the given basis are the following

$$
\begin{aligned}
& \pi_{0}=[1,0, \ldots, 0,0], \pi_{1}=[0,1, \ldots, 0,0], \\
& \ldots, \pi_{n-1}=[0,0, \ldots, 0,1] .
\end{aligned}
$$

Basis elements in the basis $\left\{\pi_{\alpha}\right\}, \alpha=\overline{0, n-1}$ possess by projection properties

$$
\pi_{\alpha} \pi_{\alpha}=\pi_{\alpha} \delta_{\alpha \beta} \sum_{\alpha=0}^{n-1} \pi_{\alpha}=1, \mathrm{z} \pi_{\alpha}=k_{\alpha} \pi_{\alpha},
$$

where $\alpha=\overline{0, n-1}$ Consequently, the set of $\mathrm{k}_{\alpha} \in \mathrm{C}$, $\alpha=\overline{0, n-1}$ is the set of eigenvalues of hypercomplex nnumber $\mathrm{z} \in \mathrm{Z}_{\mathrm{n}}$, the set of $\left\{\pi_{\alpha}\right\}, \alpha=\overline{0, n-1}$ is the eigenbasis of $\mathrm{Zn}$-algebra.

Let us remark, that the quaternion calculus can be included in the hypercomplex n-numbers' calculus being to be its particular case, since quaternions represent themselves in fact the simplest hypercomplex numbers. Really, any quaternion number $\mathrm{x}$ can be determined according to the relation

$$
x=\left(a_{1}+i a_{2}\right) e+\left(a_{3}+i a_{4}\right) j,
$$

where $\left\{\mathrm{a}_{\mathrm{m}}\right\} \in \mathrm{R}, m=\overline{1,4}$ and $\mathrm{e}, \mathrm{i}, \mathrm{j}, \mathrm{k}$ produce the basis, the elements of which are satisfying the conditions

$$
\begin{aligned}
& (i j)=k,(j i)=-k,(k i)=j,(i k)=-j, \\
& (e i)=(i e)=i,(e j)=(j e)=j,(e k)=(k e)=k .
\end{aligned}
$$

The quaternion set $\{\mathrm{Q}\}$ can be considered to be the generalization of the set $\mathrm{Zn}$ for $\mathrm{n}=2$ in accordance with the relation

$$
Z_{2}^{Q}=C_{e} \oplus C_{j},
$$

that is, it represents itself the noncom mutative ring. Geometrically, the set $\{\mathrm{Q}\}$ can be interpreted to be consisting of two mutually perpendicular hyperplanes in the space $R_{4}^{Q}$, determined by orthogonal axes - one real axis and three imaginary axes, at that, i, j, k are imaginary unities along coordinate axes in three dimensional imaginary $Z_{3}^{1}$ space, being to be the subspace of $R_{4}^{Q}$. It seems to be evident, that the space $R_{4}^{Q}$ is isomorphous to Minkowski space R4. It follows taking into account the given interpretation the conclusion, that to any quaternion number $\mathrm{x}=\left(\mathrm{a}_{1}+\mathrm{ia}_{2}\right) \mathrm{e}+\left(\mathrm{a}_{3}+\mathrm{ia}_{4}\right) \mathrm{j}$ can be set up in conformity the [2x2]-matrix according to biective mapping

$$
\begin{aligned}
& q:\left(a_{1}+i a_{2}\right) e+\left(a_{3}+i a_{4}\right) j \rightarrow \\
& {\left[\begin{array}{cc}
a_{1} & -a_{2} \\
a_{2} & a_{1}
\end{array}\right] E+\left[\begin{array}{cc}
a_{3} & -a_{4} \\
a_{4} & a_{3}
\end{array}\right] J}
\end{aligned}
$$

where $\mathrm{E}$ is [2x2] unity matrix, $\mathrm{J}$ is the following [2x2] matrix

$$
J=\left[\begin{array}{cc}
0 & 1 \\
-1 & 0
\end{array}\right]
$$

The matrices

$$
\begin{aligned}
& E=\left[\begin{array}{ll}
1 & 0 \\
0 & 1
\end{array}\right], I=\left[\begin{array}{cc}
0 & -i \\
i & 0
\end{array}\right], \\
& J=\left[\begin{array}{cc}
0 & 1 \\
-1 & 0
\end{array}\right], K=\left[\begin{array}{ll}
i & 0 \\
0 & i
\end{array}\right]
\end{aligned}
$$


produce basis for the set $\{\mathrm{x}\}$ of quaternion numbers $\{\mathrm{x}\}=$ $\left\{\left(a_{1}+i_{2}\right) e+\left(a_{3}+i a_{4}\right) j\right\}$ in the linear space of [2x2]matrices, defined over the field of real numbers.

It is evident, that the matrices (19) produce ant commutative multiplicative group. It is also understandable, that the system of quaternion numbers like to complex numbers can be constructed by an infinite number of the ways.

Let us accentuate once again, that for quantum mechanics and quantum theory at all, the relation (8) is especially significant. It allowed in the work [14] to formulate the following statement

1. Any quantum physical Hermitian operator in Hilbert space, which is set up in conformity to corresponding classical physical quantity, defines two real sets of observables, being to be connected between themselves and belonging to the field of real numbers (amplitude and phase sets or the sets of average values and their dispersion), that is, the set of complex quantities is determined by Hermitian operator in general case, which allows correctly describe the real noninstantaneous processes.

The practical consequence of the statement 1 for quantum physical tasks can be illustrated on the following example. The eigenvalues $E_{n}, n \in N$, of Hamilton operator by the solution of the main quantum mechanical equation - SchrÄodinger equation,- have to be represented in a complex form. In the application to the atom physics $\operatorname{ReE}_{n}$ represents itself the energy of the n-th atomic level [more precisely, its mathematical average], $\mathrm{ImE}_{\mathrm{n}}$ is its dispersion, that is the width of the given level. It is clear, that the usually obtained solution for the energy eigenvalues $E_{n}, n$ $\in \mathrm{N}$ by $\mathrm{E}_{\mathrm{n}} \in \mathrm{R}$ leads to the lost of the half of the possible information. The similar conclusion is true for other quantum quantities in various quantum physical tasks.

It seems to be also remarkable, that the representation of eigenvalues of Hamilton operator in the complex form is used in a practice of theoretical calculations by a number of authors to be self-evident, see, for instance, [17].

\section{Symmetry of Differential Equations and Its Connection with the Mathematical Structure of Quantum Physical Quantities}

It seems to be substantial to elucidate, whether is existing the connection between the symmetry of differential equations, describing the dynamics of the physical systems and the mathematical structure of physical quantities, incoming in the corresponding differential equations. The will show, that the way for the given elucidation is rather simple. However, we have to remark, that for the correct solution of the task formulated is essential to know the full symmetry of the differential equations, describing physical dynamic systems.

In the case of relativistic fields, in particular, in the case of electrodynamics, for instance, it have been recently found the new symmetry aspects. So, the additional gauge invariance of complex relativistic fields was studied in [15] and in [14]. It has been found, that the conserved quantity, corresponding to the invariance of generalized relativistic equations under operations of the additional gauge symmetry group - the multiplicative group $\mathrm{R}$ of all real numbers (without zero) - is the purely imaginary charge. So, it was shown, that complex fields are characterized by complex charges. Let us remember, that generalized relativistic Lagrange equations for any complex relativistic field can be represented in the form of an one matrix relativistic differential equation of the first order in partial derivatives. An analogous representation is taking place for the relativistic field equation with Hermitian conjugated (complex conjugated in the case of scalar fields) functions $u^{+}(x)=\left\{u_{i}^{+}(x)\right\}, i=\overline{1, n}$. So, the equation for the set $u(x)$ of field functions has the following form

$$
\left(\alpha_{\mu} \vartheta_{\mu}+\kappa \alpha_{0}\right) u(x)=0 .
$$

The equation for the field with Hermitian conjugated (complex conjugated in the case of scalar fields) functions is analogous

$$
\vartheta_{\mu} u^{+}(x) \alpha_{\mu}+\kappa u^{+}(x) \alpha_{0}=0
$$

The matrices $\alpha_{\mu}, \alpha_{0}$ in equations $(20,21)$ are the matrices with constant numerical elements. They have an order, which coincides with the dimension of a corresponding space of Lorentz group representation. In particular, they are $[\mathrm{n} \times \mathrm{n}]$ - matrices, if $\left\{\mathrm{u}_{\mathrm{i}}(\mathrm{x})\right\}, i=\overline{1, n}$ are scalar functions. The gauge transformation, which was found in $[14,15]$ is the following

$$
u^{\prime}(x)=\beta \exp (i \alpha) u(x),
$$

where $\alpha, \beta \in \mathrm{R}, \mathrm{u}(\mathrm{x})$ is the set of field functions. It is differed from the known gauge transformation by the presence of the factor $\beta \in \mathrm{R}$, which is substantial, since it recognizes clearly the additional symmetry of complex relativistic fields.

The following statements were proved

A. quantity - complex charge, being to be invariant under gauge transformation $u^{\prime}(x)=\beta \exp (i \alpha) u(x)$, corresponds to any complex relativistic field (scalar, vector, spinor).

B. Conserved quantity - purely imaginary charge, which is invariant under gauge transformation $u^{\prime}(x)=\beta \exp (i \alpha) u(x), \quad$ corresponds to any real relativistic field (scalar, vector, spinor).

The statement B is especially interesting. It means in fact, that in complete set of characteristic field functions of any relativistic field (complex or real) enters at least one complex (or pure imaginary) function.

It is understandable, that the transformation of field functions by the relation (22) is equivalent to the multiplication of field functions by an arbitrary complex number. The attention was drawn in [14], that the relation (22) gives the automorphism of the space of field functions. It is known, that the automorphism of any linear space leads to a number of useful properties of the objects, which belong to the given space. In particular, the consequence of the automorphic transformation of the space of EM-field functions by the relationship (22) is the following. The conservation law for charge will always take place. At the same time the energy conservation law 
and the conservation of Poynting vector will be full filled, if the given transformation is applied to EM-field potentials $[14,15]$. However, the energy conservation law and the conservation of Poynting vector, that is mathematical constructions, to which enter $\vec{E}-, \vec{H}$ - vector functions, will not be full filled by the transformation (22) at an arbitrary $\beta$. The charge remains to be Lorentz invariant quantity [14], at the same time both the field characteristics, the energy and the impulse (determined by Poynting vector) are not Lorentz invariant quantities [14]. It is remarkable, that the conclusion on the charge Lorentz invariance was formulated in [19] to be self-evident. It was concluded in [14], that the charge conservation law for EM-field can be considered to be more fundamental, since it is full filled even through the energy and the impulse conservation laws do not take place.

Both the statements give the key for the correct generalization of relativistic field equations, in particular, electrodynamics equations.

Further, the differential equations (20) and (21) are invariant under the same transformation (22). Therefore, it is seen, that the correspondence between the symmetry of differential equations and the mathematical nature [in the concept of the number theory] of the quantities, incoming in given equations seems to be taking place.

Given suggestion can be proved and generalized.

So, we come to the statement:

2. Differential equations, which are invariant under transformations of groups, which are symmetry groups of mathematical numbers (considered within the frames of the number theory) determine the mathematical nature of the quantities, incoming in given equations.

In the case of the invariance of differential equations under transformation (22), that is, in the case of the invariance under transformations of multiplicative group of the linear space of complex numbers over the field of real numbers, the proof seems to be evident. Really, the possibility of multiplication of full set of field functions on complex number means, that the field functions themselves have to be complex-defined functions.

It seems to be substantial that differential equations for the dynamics of the nonrelativistic classical mechanics are invariant under transformation (22) too. Really, the dynamics of classical mechanics systems is described by Lagrange equations or by equivalent canonical Hamilton equations. It is known, that, for instance, Hamilton equations are invariant under contact transformations of variables, that is, under the transformation of linear elements- positions and directions, but not the points' transformation. The transformation (22) is referred to the contact transformations' class. It means, that all physical quantities, which determine the dynamics of classical mechanics systems have to be represented by a quantummechanical description by one of the variants of the representation of complex number, in particular, taking into account (8), by Hermitian matrices. Therefore, we have the proof of the statement, being to be the proof of the main postulate of quantum mechanics.

3. To any mechanical quantity can be set up in the correspondence the Hermitian matrix by the quantization.

It is in fact the consequence of the statement 2. The choose of the construction of the mathemaical apparatus of quantum mechanics on the base of Hermitian matrices is convenient, however, it is the only one variant from the infinity of variants of the representations of quantum mechanical quantities by complex numbers. The given conclusion opens the high functional possibilities for the development of the quantum theory.

We have to remark, that the description of the processes in classical mechanics by using of complex number is also correct. But in very many practical cases, for instance, for mechanical tasks, described by Newton equation, forces and impulses can be characterized by the same phase factor (that is, it can be not taking into consideration).

It will be considered father the case of the invariance of differential equations under transformations of the anticommutative multiplicative group of the quaternion ring.

It is the case of the electrodynamics, for which the following statement takes place

4. To any electrodynamics quantity can be set up in the correspondence the quaternion matrix by the quantization of EM-field.

Let us prove the invariance of Maxwell equations under transformations (22) independently on the symmetry studies of Maxwell equations in [14], which gave the new insight on the nature of electromagnetic field quantities. They have in general case the quaternion structure, consisting of four independent constituents, which differ from each other by the parities under space inversion and time reversal. The presence along with vector quaternion characteristics the independent scalar quaternion characterics of EM-field established in [14] allows to describe EM-field instead of unobservable vector and scalar potentials by the observable electric field 4-vectorfunction with the components $E_{\alpha}(\vec{r}, t)=\left\{E_{x}(\vec{r}, t), E_{y}(\vec{r}, t), E_{z}(\vec{r}, t), i \frac{c \rho_{e}(\vec{r}, t)}{\lambda}\right\}$ and

(or in the case of free EM-field) by means of the magnetic field 4-vector-function

$H_{\mu}(\vec{r}, t)=\left\{H_{x}(\vec{r}, t), H_{y}(\vec{r}, t), H_{z}(\vec{r}, t), i \frac{c \rho_{m}(\vec{r}, t)}{\lambda}\right\}$, where ic $\rho_{e}(\vec{r}, t)$, ic $\rho_{m}(\vec{r}, t)$ are the $j_{4}(\vec{r}, t)$-component of 4-current density, corresponding to the contribution of an electric and a magnetic component of charge densities correspondingly, is conductivity, which for the case of an EM-field propagation in the vacuum is $\lambda_{v}=\frac{1}{120 \pi}(\mathrm{Ohm})^{-1}$.

The electric $\vec{j}_{e}(\vec{r}, t)$ (and magnetic $\vec{j}_{g}(\vec{r}, t)$ in the general case) current densities in the right side of Maxwell equations can be represented by well known relations $\vec{j}_{e}(\vec{r}, t)=\rho_{e}(\vec{r}, t) \vec{v}_{e}(\vec{r}, t)$, where $\vec{v}_{e}(\vec{r}, t)$ is the charge velocity in a joint system \{EM-field + matter $\}$ and by the analogous relation (in the general case) for a magnetic $\vec{j}_{g}(\vec{r}, t)$ current density. Therefore, in both the parts of Maxwell equations all the quantities are expressed through the full set of EM-field functions. Now, the invariance of Maxwell equations under transformations of the multiplicative group of the quaternion ring becomes to be evident, since the multiplication of all the EM-field 
functions on the same quaternion from the right (or from the left) remains the Maxwell equations to be invariant.

Therefore, take into account the statement 4 , we really obtain the independent proof that all EM-field quantities have to be considered to be quaternions in the general case. We have to remark, that in the practice of theoretical considerations and the treatment of experimental results in the tasks with the participation of EM-field the complex EM-field quantities are usually used [16] for the correct description of electromagnetic phenomena in the correspondence with the symmetry of the Maxwell equations under the multiplicative group of the linear space of complex numbers over the field of real numbers. The given picture is practically always used both in the theory and in the applications. However, even the given representation in some cases is insuecient. It is concerned, for instance, the dynamics of optical transitions [14].

So, the invariance of Maxwell equations under transformations of the quaternion non-abelian multiplicative group leads to the conclusion, that really to any electrodynamics quantity can be set up in the correspondence the quaternion matrix by the quantization of EM-field. It is in fact the consequence of the presence along with the symmetry of Maxwell equations under transformations, given by (22), the Rainich [18] dual symmetry and the additional dual symmetry, established in [14].

Let us remark for a comparison, that the equations of the dynamics of mechanical systems are not invariant under transformations of the quaternion multiplicative group. It is the consequence of the non-abelian character of the given group. The given conclusion seems to be significant. It means in particular that quantum mechanics in distinction from quantum electrodynamics cannot be formulated within the frames of the quaternion theory immediately. At the same time, there are at present a number of publications, devoted just to the reformulation of quantum mechanics on the quaternion base, for instance, $[20,21,22,23]$. Moreover, they are very popular. Let us give the concrete example of the incorrectness of the given re-formulation. The author of $[20,21,22,23]$ has claimed on the derivation of a "universal quantum wave equation" that yields Dirac, Klein-Gordon, SchrÄodinger and quantum heat equations. However, the author itself indicates that the additional transformations are needed to agree so colled "universal quantum equation" with Dirac equation. They are the following: replacing the particle mass $\mathrm{m}_{0}$ by $\mathrm{im}_{0}$, or changing space and time coordinates by it and $\overrightarrow{i r}$, respectively. The physical nature of the given replacement is not explained by the author and it seems to be evident, that it cannot be understandable in the principle. The appearance of the imaginary unity in a number of expressions in the papers above cited leads to very incorrect physical conclusions. It is mathematical consequence of the non-abelian character of the multiplicative group of quaternions. The resemblance of the form of" universal quantum wave equation" and Dirac form, or Klein-Gordon equations derived from the given equation can be explained by the minimal deviation of the non-abelian character of the multiplicative group of quaternions from the abelian groups, it is the only ant commutative.

An attempt of a new formulation of quantum mechanics based on differential commutator brackets is undertaken in
[24]. Differential commutator brackets were introduced in [25]. The authors of $[24,25]$ have used the following relations

$$
\left[\frac{\partial}{\partial t}, \nabla\right]=0,\left[\frac{\partial}{\partial t}, \nabla\right] \cdot=0,\left[\frac{\partial}{\partial t}, \nabla\right] \times=0,
$$

which seems to be correct in the application to scalar or vector wave functions. However, they have used in the subsequent consideration the quaternion wave function, which is inapplicable immediately for the description of quantum mechanics systems.

So, from the given consideration emerges the task: is it possible to built quantum mechanics on the quaternion base, or not. We know, taking into account the conclusions in the Section 2, that there is an infinite number of the ways to build the quantum theory from the corresponding classical theory, which corresponds to an infinite number of the ways to build the complex numbers in a matrix form. On the other hand, there is the connection between quaternions and complex numbers above considered. It becomes then clear, that the new basis for quaternion set has to be choosed, which will be simultaneously the basis of complex numbers. Given task is correct. Really, let us designate the quaternion set by $\{\mathrm{Q}\}$

$$
\{Q\}=\left\{Q=a_{0} e+a_{1} i+a_{2} i+a_{3} k \mid a_{0}, a_{1}, a_{2}, a_{3} \in R\right\} .
$$

$\{\mathrm{Q}\}$ is the non-abelian division ring and it forms a 4dimensional normed division algebra over the real numbers R. Algebras of the real numbers R and complex numbers $C$ are sub algebras of the $\{Q\}$ algebra. Let us designate the quaternion conjugate of $\mathrm{Q}=\mathrm{a}_{0} e+\mathrm{a}_{1} i+\mathrm{a}_{2} j+\mathrm{a}_{3} k$ by $\mathrm{Q}^{*}$. Furthermore, we will define ReQ $:=\mathrm{a}_{0}$ and $\vec{Q}:=\mathrm{a}_{1} i+\mathrm{a}_{2} j+\mathrm{a}_{3} k$, that is, the usually used biquaternion form will be considered. To obtain the quaternion division ring with a 4-dimensional normed division algebra over the real numbers $\mathrm{R}$ and with the basis, which is simultaneously the basis of complex numbers, it is sufficient, for example, for the quaternion basis $\{\mathrm{e}, \mathrm{i}, \mathrm{j}, \mathrm{k}\}$ to set in the correspondence the matrices, determined by the following bijective mappings

$$
\begin{aligned}
& e: \rightarrow E\left[\begin{array}{ll}
1 & 0 \\
0 & 1
\end{array}\right], i: \rightarrow I^{\prime}=i \sigma_{z}=\left[\begin{array}{cc}
i & 0 \\
0 & -i
\end{array}\right], \\
& j: J^{\prime}=i \sigma_{y}=\left[\begin{array}{cc}
0 & 1 \\
-1 & 0
\end{array}\right], k: \rightarrow i \sigma_{x}=K^{\prime}=\left[\begin{array}{ll}
0 & i \\
i & 0
\end{array}\right],
\end{aligned}
$$

where $\sigma_{x}, \sigma_{y}, \sigma_{z}$ are Pauli matrices. It is easily to see, that the matrices $\mathrm{E}$ and -iJ' produce basis of complex numbers in the quaternion set $\{\widehat{Q}\}$. There is the biective mapping between quaternion set $\{Q\}$ and the new quaternion set $\{\hat{Q}\}$ with basis, determined by (25).

$$
\{Q\}: \rightarrow\{\widehat{Q}\}: \rightarrow\{[\hat{Q}]\}=\left\{\left[\begin{array}{cc}
a_{0} e+i a_{1} & a_{2}+i a_{3} \\
-a_{2}+i a_{3} & a_{0} e-i a_{1}
\end{array}\right]\right\},
$$

where $\{\widehat{Q}\}$ is the matrix representation of quaternion set $\{\widehat{Q}\}$, which belongs simultaneously to the representation of complex numbers $\mathrm{C}^{2 \times 2}$. The quaternion conjugates 
$\left\{\widehat{Q}^{*}\right\}$ in the given representation are the Hermitian conjugates of the matrices, determined by (26), that is $\left\{\widehat{Q}^{*}\right\}=\{[\widehat{Q}]+\}$. The quaternion norm is the square root of the determinant

$$
\|\hat{Q}\|=\sqrt{\operatorname{det}[\hat{Q}]}
$$

Mapping, given by (26), realizes the connection between quaternions and operators on Hilbert spaces.

Let us remark, that, if the quaternions are defined by equations (14) - (16), then it is more convenient to realize the biective mapping, similar to mapping (26) by means of the matrices E, I, J, K, determined by relations (19), at that all the quaternion matrices have to be represented through the basis matrices of complex numbers. It is the second way of the correct formulation of quantum mechanics on the quaternion base.

Therefore, to quaternion numbers can be set up in the correspondence the complex numbers, matrix representations of which is choosed by two ways above indicated, that is, two concrete representations are choosed from the infinite sets of representation forms. It, in its turn, means, that the differential equations, which describe the dynamics of mechanical systems, will be invariant under multiplicative group of the complex numbers, obtained in given two ways and, consequently, transformed sets of quaternion numbers can be used to build the new two versions of quantum mechanics.

So, we have obtained the strict proof, that direct application of quaternions with usually used basis $\{e, i, j$, $\mathrm{k}$ \} to build the new version of quantum mechanics is incorrect and we have found the ways for the creation of the correct versions of quantum mechanics on the quaternion base.

We wish also to report that recently [26] the conclusion on the status of the second main postulate of quantum mechanics was presented. The strict mathematical proof of the hypothesis of SchrÄodinger on the existence of the field scalar function, being to be observable quantity, just charge density was obtained. It was simultaneously shown, that the SchrÄodinger equation describes corpuscular aspect of dual corpuscular-wave characteristics of the state of atomic systems. Just, the field scalar function, being to be the solution of the corresponding no stationary in general case SchrÄodinger equation and being to be the function the only of coordinates and time is responsible for the corpuscular aspect description.

The atomic system in [26] is considered from the positions of the field theory, that is, it seems to be the association of elementary particles with corresponding fields. The given fields being to be the media for the particles' propagation are responsible for the wave aspect in the characteristics of atomic systems. Moreover, it was accentuated, that the field scalar function actually describes the state of the corpuscles in the dual particlefield atomic system, since the full set of the functions for the description of EM-field is consisting of four scalar functions or equivalently, from one scalar and one vector functions. The information, which is given by an observable vector function of EM-field - field strength function is included in an implicit form in SchrÄodinger equation for the field scalar function, that is, it is taking into consideration too.

The wave aspect is described by the independent scalar wave function which was also introduced in quantum physics by SchrÄodinger. The description of the wave aspect was developed in the work [26]. The expression for the scalar wave function, based on the boson Fermi liquid model of EM-field was derived. It represents itself the superposition of Bloch-like waves, being to be mathematically identical to Bloch waves in matter single crystals.

The attention is drawn, that the second main postulate being to be mathematically strictly grounded in the SchrÄodinger formulation of quantum mechanics, in the popular probabilistic form of quantum mechanics cannot be proved in the general case, although the probabilistic theatise, proposed by Born is true in a number of special cases, at that, the given cases embrace the wide range of quantum physics phenomena.

It is also indicated, that in order to obtain a new information on the properties of the quantum systems the versions of quantum mechanics which can describe the single events have to be elaborated. For the given aim can be useful the modern achievements in the mathematics, in particular, in operator theory, for instance, the results reported in the works $[27,28,29]$. It is taking into account that operator calculus is based calculus for the quantum theory.

\section{Conclusions}

The symmetry study of main differential equations of mechanics and electrodynamics has shown, that differential equations, which are invariant under transformations of groups, which are symmetry groups of mathematical numbers (considered within the frames of the number theory) determine the mathematical nature of the quantities, incoming in given equations.

The main postulate of quantum mechanics, consisting in that, that to any mechanical quantity can be set up into the correspondence the Hermitian matrix by the quantization, was proved.

The high symmetry of Maxwell equations, consisting in the presence along with the symmetry under transformations, given by (22), the Rainich dual symmetry and the additional hyperbolic dual symmetry, established in [14], allowed to show, that to EM-field functions, incoming in given equations, can be set up into the correspondence the quaternion matrices by their quantization.

It is concluded, that the equations of the dynamics of mechanical systems are not invariant under transformations of the multiplicative group of the quaternion ring and, consequently, the direct application of quaternions with the usually used basis $\{\mathrm{e}, \mathrm{i}, \mathrm{j}, \mathrm{k}\}$ to build the new version of quantum mechanics is incorrect. It is the con-sequence of the nonabelian character of the given group.

At the same time, we have found the correct ways for the creation of the new versions of quantum mechanics on the quaternion base by means of the choice of the representation of the quaternion matrices through the basis matrices corresponding to complex numbers, then the 
equations of the dynamics of quantum mechanical systems will be invariant under the multiplicative group of the field of complex numbers.

\section{Reference}

[1] Landau L.D, Lifshitz E.M, Quantum Mechanics, Moscow, Nauka, 1989, 768 pp.

[2] Kramers H A, Quantum Mechanics, North-Holland, Am- sterdam, 1958.

[3] Bohm A, Quantum Mechanics: Foundations and Ap- plications, Springer-Verlag, N.-Y., Berlin, Heidelberg, Tokyo, 1986, M., Mir, 1990, 720 pp.

[4] Davydov A.S, Quantum Mechanics, Moscow, Nauka, 1973, 703 pp.

[5] Heisenberg W, Quantum-Theoretical Re-Interpretation of Kinematic and Mechanical Relations, Zeitschrift fu"r Physik, 33 (1925) 879-893.

[6] Born M, Jordan P, Zur Quantenmechanik, Zeitschrift für Physik, 34 (1925) 858-888.

[7] Born M, Heisenberg W, Jordan P, Zur Quantenmechanik II, Zeitschrift fu"r Physik, 35 (1926) 557-606.

[8] Dirac P.A.M, The Fundamental Equations of Quantum Mechanics, Proc. Roy. Soc. A 109 (1925) 642-653.

[9] Pauli M,U* ber das Wassestoffspektrum vom Standpunkt der neuen Quantummechanik, Zeitschrift für Physik, 36 (1926) 336363.

[10] Dirac P.A.M, Quantum Mechanics and a Preliminary Investigation of the Hydrgen Atom, Proc. Roy. Soc. A, 110 (1926) 561-569

[11] Dmitri Yerchuck, Alla Dovlatova, Andrey Alexandrov, Symmetry of Differential Equations and Quantum The- ory, 2nd International Conference on Mathematical Mod- eling in Physical Sciences, 1-5 September, 2013, Prague, Czechia, Journal of Physics: Conference Series, 490 (2014) 012233-7.

[12] Dovlatova A, Yearchuck D, QED Model of Resonance Phenomena in Quasionedimensional Multichain Qubit Systems, Chem. Phys. Lett., 511 (2011) 151-155.

[13] Yerchuck D, Dovlatova A, Quantum Optics Effects in Quasi-OneDimensional and Two-Dimensional Carbon Materials, Journal of Physical Chemistry C, 116, N 1 (2012) 63-80.
[14] A.Dovlatova and D.Yerchuck, Concept of Fully Du- ally Symmetric Electrodynamics, 7th International Con- ference on Quantum Theory and Symmetries (QTS7), J.Physics: Conference Series, 343 (2012) 012133, 23 pp.

[15] D.Yearchuck, A.Alexandrov and A.Dovlatova, To Nature of Electromagnetic Field, Appl. Math. Comput. Sci., 3, N 2 (2011) 169-200.

[16] Andre Angot, Complements de Mathematiques, Paris, 1957, 778

[17] Baryshevsky V.G, Time-Reversal-Violating Optical Gy- rotropy, arXiv: hep-ph/9912438v3 23 Feb 2000.

[18] Rainich G Y, Electrodynamics in the General Relativity Theory, Trans. Am. Math. Soc., 27 (1925) 106-136.

[19] Strazhev V.I, Tomilchick L.M, Electrodynamics with Magnetic Charge, Minsk, Nauka i Tekhnika, 1975, 336 pp.

[20] Arbab A. I., The Unified Quantum Wave Equation, Hadronic Journal, 33, (2010) 707-716.

[21] Arbab A. I., Derivation of Dirac, Klein-Gordon, Schr“odinger, Diffusion and Quantum Heat Transport Equations from a Universal Quantum Wave Equation, EPL, 92 (2010) 40001.

[22] Arbab A. I., The Quaternionic Quantum Mechanics, Ap- plied Physics Research, 3, N 2 (2011) 160-170.

[23] Arbab A. I., Phys.Essay, 24 (2011) 254.

[24] Arbab A. I., Yassein F. A., A New Formulation of Quan- tum Mechanics, Journal of Modern Physics, 3 (2012) 163-169.

[25] Arbab A. I., Yassein F. A., A New Formulation of Electrodynamics, Journal of Electromagnetic Analysis and Applications, 2, N 8 (2010) 457.

[26] Dmitri Yerchuck, Alla Dovlatova, Felix Borovik, Yauhen Yerchak, Vyacheslav Stelmakh, To Principles of Quan- tum Mechanics Development, International Journal of Physics, 2, N 5 (2014) 129145.

[27] V.N. Mishra, K. Khatri, L.N. Mishra, On SimultaneousApproximation for Baskakov-Durrmeyer-Stancu type op- erators, Journal of Ultra Scientist of Physical Sciences, 24, N (3)A, (2012) 567-577.

[28] V.N. Mishra, H.H. Khan, K. Khatri, L.N. Mishra, Hypergeometric Representation for Baskakov-Durrmeyer- Stancu Type Operators, Bulletin of Mathematical Analy- sis and Applications, 5, N 3 (2013) 18-26.

[29] Prashantkumar Patel, and Vishnu Narayan Mishra, Rate of Convergence of Modified Baskakov-Durrmeyer Type Operators for Functions of Bounded Variation, Journal of Difference Equations, Volume 2014, Article ID 235480, 6 pages. 